\title{
PLANILHA ELETRÔNICA PARA ESTIMATIVAS DE FUGACIDADES USANDO EQUAÇÃO DE LEE-KESLER
}

\author{
J. S. da SILVA NETO ${ }^{1}$, F. P. FELINTO ${ }^{1}$, W. D. N. A. dos REIS ${ }^{1}$, J. A. CAVALCANTE ${ }^{1}$ e N. A. \\ $\operatorname{COSTA}^{1}$ \\ ${ }^{1}$ Universidade Federal da Paraíba \\ E-mail: nagelalves@hotmail.com
}

\begin{abstract}
RESUMO - O presente trabalho tem por objetivo apresentar uma planilha eletrônica para as estimativas de fugacidades e coeficientes de fugacidades de espécies puras, de misturas e de espécies $i$ na mistura através da equação não analítica de Lee-Kesler. O trabalho é dividido em cinco partes: a) criação do banco de dados contendo propriedades críticas e constantes características do fluido simples e do fluido de referência; b) criação de caixas de diálogos para interação do usuário com o programa, c) codificação de subprograma VBA para estimativas das propriedades volumétricas, d) codificações VBA para estimativas das fugacidades e e) validação dos resultados fornecidos pelo programa. $\mathrm{O}$ resultado final é um aplicativo, para fins educacionais e/ou comerciais, capaz de fornecer estimativas das propriedades volumétricas e fugacidades com rapidez e confiabilidade.
\end{abstract}

\section{INTRODUÇÃO}

As propriedades volumétricas de substância pura e de misturas são estimadas através de procedimentos análogos (para misturas, os parâmetros são expressos como funções das frações molares). As expressões são resumidas no Quadro 1.

Quadro 1 - Resumo das expressões fornecidas pela equação de Lee-Kesler

- Fluido simples $(k=0)$ e fluido de referência $(k=R)$ :
$Z^{(k)}=1+\frac{B^{(k)}}{V_{r}^{(k)}}+\frac{C^{(k)}}{V_{r}^{(k)^{2}}}+\frac{D^{(k)}}{V_{r}^{(k)^{5}}}+\frac{c_{4}^{(k)}}{T_{r}^{3} V_{r}^{(k)^{2}}}\left(\beta^{(k)}+\frac{\gamma^{(k)}}{V_{r}^{(k)^{2}}}\right) \mathrm{e}^{-\gamma^{(k)} / V_{r}^{(k)^{2}}},\left(V_{r}\right)^{(k)}=\frac{Z^{(k)} T_{r}}{P_{r}}$ e $\left(\rho_{r}\right)^{(k)}=\frac{1}{\left(V_{r}\right)^{(k)}}$
$(\ln \phi)^{(k)}=\frac{B^{(k)}}{V_{r}^{(k)}}+\frac{C^{(k)}}{2\left(V_{r}^{(k)}\right)^{2}}+\frac{D^{(k)}}{5\left(V_{r}^{(k)}\right)^{5}}+E^{(k)}+\left(Z^{(k)}-1\right)-\ln Z^{(k)}$
o Parâmetro auxiliar: $E^{(k)}=\left(\frac{C_{4}^{(k)}}{2 \gamma^{(k)} T_{r}^{3}}\right)\left[\left(\beta^{(k)}+1\right)-\left(\beta^{(k)}+1+\frac{\gamma^{(k)}}{\left(V_{r}^{2}\right)^{(k)}}\right) e^{-\gamma^{(k)} /\left(v_{r}^{2}\right)^{(k)}}\right]$
o Entalpia residual da mistura: $\frac{H^{R}}{R T}=\Gamma=\Gamma^{(0)}+w_{m} \Gamma^{(1)} \mathrm{e} \Gamma^{(1)}=\frac{\Gamma^{(R)}-\Gamma^{(0)}}{w^{(R)}}$


$\Gamma^{(k)}=-\frac{b_{2}^{(k)}+2 b_{3}^{(k)} / T_{r}+3 b_{4}^{(k)} / T_{r}^{2}}{T_{r}\left(V_{r}\right)^{(k)}}-\frac{c_{2}^{(k)}-3 c_{3}^{(k)} / T_{r}^{2}}{2 T_{r}\left(V_{r}^{2}\right)^{(k)}}+\frac{d_{2}^{(k)}}{5 T_{r}\left(V_{r}^{5}\right)^{(k)}}+3 E^{(k)}+\left(Z^{R}\right)^{(k)}$

- Correção: $Z^{(1)}=\frac{Z^{(R)}-Z^{(0)}}{w^{(R)}},\left(V_{r}\right)^{(1)}=\frac{\left(V_{r}\right)^{(R)}-\left(V_{r}\right)^{(0)}}{w^{(R)}}$ e $(\ln \phi)^{(1)}=\frac{(\ln \phi)^{(R)}-(\ln \phi)^{(0)}}{w^{(R)}}$

- Fluido de interesse: $Z=Z^{(0)}+w Z^{(1)}, V_{r}=\left(V_{r}\right)^{(0)}+w\left(V_{r}\right)^{(1)} \mathrm{e} \ln \phi=(\ln \phi)^{(0)}+w(\ln \phi)^{(1)}$

Os parâmetros $B, C$ e $D$ são descritos no Quadro 2.

Quadro 2 - Parâmetros da equação BWR modificada

$$
\begin{array}{c|c|c}
B=b_{1}-\frac{b_{2}}{T_{r}}-\frac{b_{3}}{T_{r}^{2}}-\frac{b_{4}}{T_{r}^{3}} & C=c_{1}-\frac{c_{2}}{T_{r}}+\frac{c_{3}}{T_{r}^{3}} \quad D=d_{1}+\frac{d_{2}}{T_{r}}
\end{array}
$$

Lee e Kesler (1975) determinaram as constantes características da equação BWR modificada através da regressão de um conjunto de dados experimentais. Os pesquisadores utilizaram as seguintes restrições: a) critério de equilíbrio entre fases, $f_{i}^{V}=f_{i}^{L}$, onde $f_{i}^{V}$ e $f_{i}^{L}$ são, respectivamente, as fugacidades das fases vapor e líquida e b) condição de estabilidade do ponto crítico, $\left(\partial P_{r} / \partial V_{r}\right)_{T r}=\left(\partial^{2} P_{r} / \partial V_{r}^{2}\right)_{T_{r}}=0$. Os resultados reportados são mostrados na Tabela 1.

Tabela 1 - Constantes ajustadas para os fluidos simples e de referência

\begin{tabular}{lcc|lcc}
\hline Constantes & $\begin{array}{c}\text { Fluido } \\
\text { Simples }\end{array}$ & $\begin{array}{c}\text { Fluido } \\
\text { Referência }\end{array}$ & Constantes & $\begin{array}{c}\text { Fluido } \\
\text { Simples }\end{array}$ & $\begin{array}{c}\text { Fluido } \\
\text { Referência }\end{array}$ \\
\hline$b_{1}$ & 0,1181193 & 0,2026579 & $c_{3}$ & 0,000000 & 0,0169010 \\
$b_{2}$ & 0,2657280 & 0,3315110 & $c_{4}$ & 0,042724 & 0,0415770 \\
$b_{3}$ & 0,1547900 & 0,0276550 & $d_{1} \times 10^{4}$ & 0,155488 & 0,4873600 \\
$b_{4}$ & 0,0303230 & 0,2034880 & $d_{2} \times 10^{4}$ & 0,623689 & 0,0740336 \\
$c_{1}$ & 0,0236744 & 0,0313385 & $\beta$ & 0,653920 & 1,2260000 \\
$c_{2}$ & 0,0186984 & 0,0503618 & $\gamma$ & 0,060167 & 0,0375400 \\
\hline
\end{tabular}

Lee e Kesler (1975) recomendam que o fator acêntrico da espécie pura seja estimado através da seguinte expressão:

$$
w_{i}=\frac{\ln P_{b r i}^{s a t}-\left.f^{(0)}\right|_{T_{b r i}}}{\left.f^{(1)}\right|_{T_{b r i}}}
$$

onde $P_{b r i}^{\text {sat }}$ é a pressão de vapor reduzida no ponto normal de ebulição da espécie $i$, $\left.f^{(0)}\right|_{T_{b r}}=A^{(0)}-\frac{B^{(0)}}{T_{b r}}+C^{(0)} \ln T_{b r}+D^{(0)} T_{b r}^{6}$ e $\left.f^{(1)}\right|_{T_{b r}}=A^{(1)}-\frac{B^{(1)}}{T_{b r}}+C^{(1)} \ln T_{b r}+D^{(1)} T_{b r}^{6}$. 
O conjunto de regra de mistura recomendado por Reid et al. (1988) para a equação de estado de Lee-Kesler é apresentado no Quadro 3. Plocker et al. (1978) fornecem os valores dos parâmetros de interação binária para 150 sistemas binários.

Quadro 3 - Regras de mistura de Lee-Kesler

$$
\begin{array}{ccc}
V_{c l s}=\frac{\left(V_{c l}^{1 / 3}+V_{c s}^{1 / 3}\right)^{3}}{8} \quad Z_{c m}=0,2905-0,085 w_{m} \quad w_{m}=\sum_{l=1}^{N} y_{l} w_{l} & V_{c m}=\sum_{l=1}^{N} \sum_{s=1}^{N} y_{l} y_{s} V_{c l s} \\
T_{c m}=\frac{1}{V_{c m}^{\eta} \sum_{l=1}^{N} \sum_{s=1}^{N} y_{l} y_{s} V_{c l s}^{\eta} T_{c l s}} \quad P_{c m}=\frac{Z_{c m} R T_{c m}}{V_{c m}} & T_{c l s}=k_{l s} \sqrt{T_{c l} T_{c s}}
\end{array}
$$

Formulação original Lee e Kesler (1975): $\eta=1$ e $k_{l s}=1$ para $l, s=1,2, \ldots, N$.

Formulação de Plocker et al. (1978): $\eta=0,25$ e $k_{l s}$ são obtidos a partir da regressão de dados experimentais

O resumo das expressões para as estimativas das propriedades e suas derivadas é apresentado no Quadro 4. Os detalhes da modelagem matemática são reportados por Costa (2014).

Quadro 4 - Propriedades e suas derivadas

o Coeficiente de fugacidade da espécie $i$ na mistura:

$$
\ln \hat{\phi}_{i}=\ln \phi-\sum_{j \neq i} y_{j}\left[\left(\frac{\Gamma}{T_{c m}}\right)\left(\frac{\partial T_{c m}}{\partial y_{j}}\right)_{\alpha}-\left(\frac{Z^{R}}{P_{c m}}\right)\left(\frac{\partial P_{c m}}{\partial y_{j}}\right)_{\alpha}+(\ln \phi)^{(1)}\left(\frac{\partial w_{m}}{\partial y_{j}}\right)_{\alpha}\right]
$$

o Derivadas parciais:

$$
\begin{aligned}
& \left(\frac{\partial w_{m}}{\partial y_{j}}\right)_{T, P, y_{k \neq i, j}}=w_{j}-w_{i},\left(\frac{\partial V_{c m}}{\partial y_{j}}\right)_{T, P, y_{k \neq i, j}}=2 \sum_{l=1}^{N} y_{l}\left(V_{c l j}-V_{c l i}\right) \\
& \left(\frac{\partial T_{c m}}{\partial y_{j}}\right)_{T, P, y_{k \neq i, j}}=\frac{2 \sum_{l=1}^{N} y_{l}\left(V_{c l j}^{\eta} T_{c l j}-V_{c l i}^{\eta} T_{c l i}\right)}{V_{c m}^{\eta}}-\frac{2 \eta T_{c m} \sum_{l=1}^{N} y_{l}\left(V_{c l j}-V_{c l i}\right)}{V_{c m}} \\
& \left(\frac{\partial P_{c m}}{\partial y_{j}}\right)_{T, P, y_{k \neq i, j}}=P_{c m}\left[\left(\frac{1}{Z_{c m}}\right)\left(\frac{\partial Z_{c m}}{\partial y_{j}}\right)_{\alpha}+\left(\frac{1}{T_{c m}}\right)\left(\frac{\partial T_{c m}}{\partial y_{j}}\right)_{\alpha}-\left(\frac{1}{V_{c m}}\right)\left(\frac{\partial V_{c m}}{\partial y_{j}}\right)_{\alpha}\right] \\
& \left(\frac{\partial Z_{c m}}{\partial y_{j}}\right)_{T, P, y_{k \neq i, j}}=-0,085\left(w_{j}-w_{i}\right)
\end{aligned}
$$




\section{METODOLOGIA}

A metodologia empregada para o desenvolvimento do aplicativo EXCEL/VBA é apresentada na Figura 1.

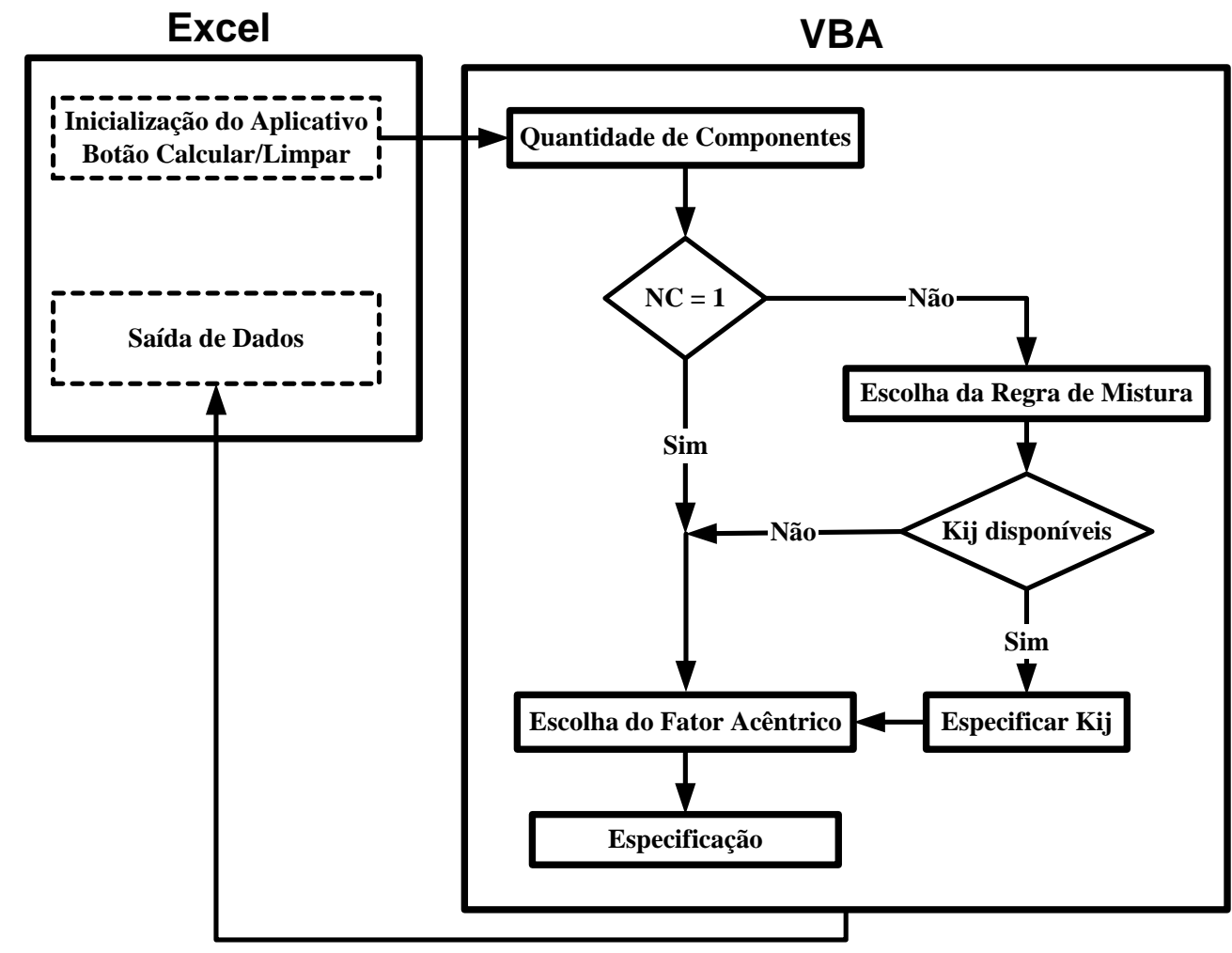

Figura 1 - Esquema do desenvolvimento do aplicativo

\subsection{Etapas do Desenvolvimento do Aplicativo}

As etapas do desenvolvimento do aplicativo EXCEL/VBA são descritas nos tópicos seguintes. Para demonstrar o aplicativo criado, considere uma mistura equimolar constituído de metiletilcetona (1) - tolueno (2) a $25 \mathrm{kPa}$ e $420 \mathrm{~K}$ reportada por Assael et al. (1998).

Banco de dados: O banco de dados contém as propriedades físicas de 41 espécies químicas (massa molar, temperatura normal de ebulição, temperatura crítica, pressão crítica, volume molar crítico, fator de compressibilidade crítico, fator acêntrico e momento dipolo). Três conjuntos de propriedades físicas (PF) foram colocados no banco de dados: PF1 - reportadas por Reid et al. (1988); PF2 - reportadas por Poling et al. (2000) e PF3 - reportadas por Smith et al. (2007).

Quantidade de espécies químicas: A caixa de diálogo para a escolha da quantidade de espécies presentes no sistema é mostrada na Figura 2. O usuário pode escolher na faixa de 1 a 12 espécies. 
Escolha de espécies químicas: A caixa de diálogo para a escolha das espécies presentes é mostrada na Figura 3.

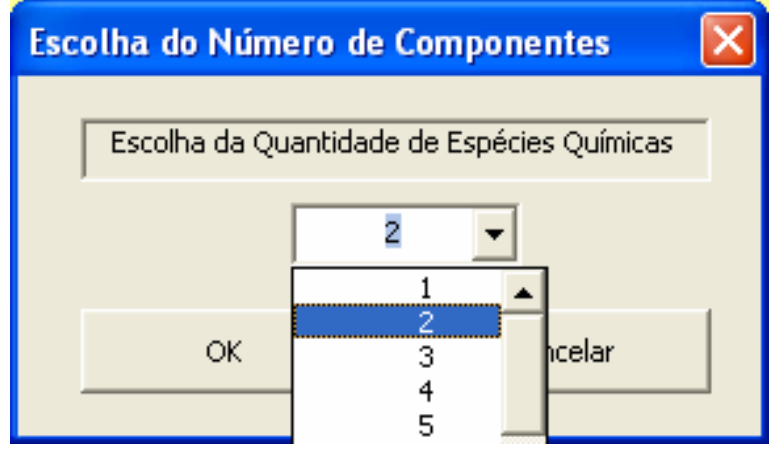

Figura 2 - Escolha da quantidade de espécies

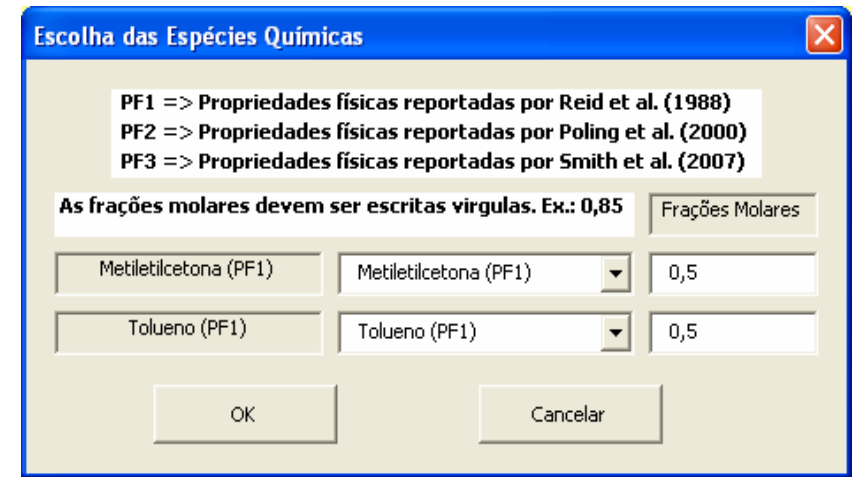

Figura 3 - Escolha das espécies Químicas

Escolha do fator acêntrico: As opções do usuário são duas: a) fator acêntrico recomendado por Lee e Kesler e b) Fornecidos na literatura PF1, PF2 e PF3.

Escolha da regra de mistura: Se a quantidade de espécie for diferente de um, o aplicativo abre a caixa de diálogo para a escolha da regra de mistura: a) Lee e Kesler (1975) e b) Plocker et al. (1978).

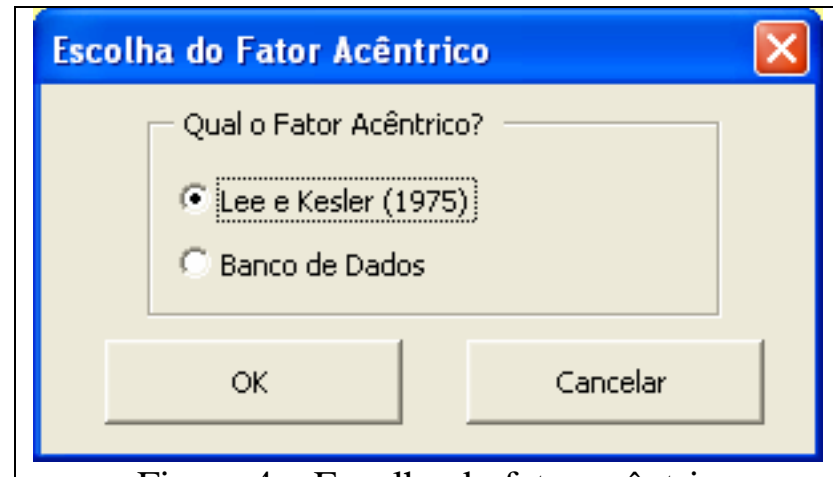

Figura 4 - Escolha do fator acêntrico

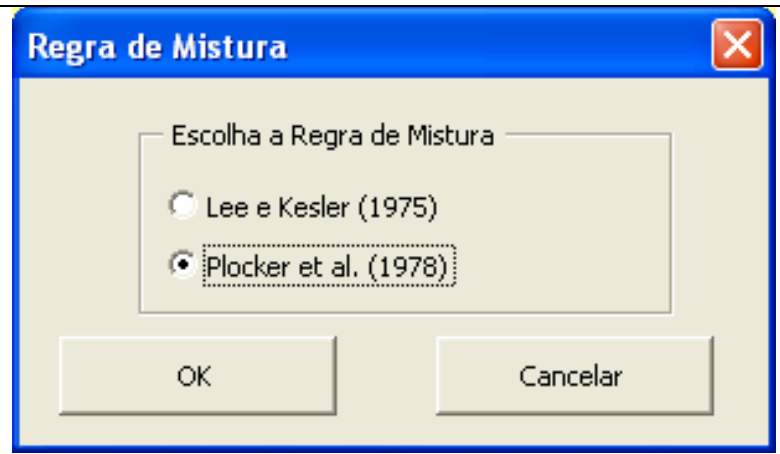

Figura 5 - Escolha da regra de mistura

Na opção Lee e Kesler (1975), todos os parâmetros binários são tomados como sendo unitários. Se a escolha for a regra de mistura de Plocker et al. (1978), o aplicativo abre a caixa de diálogo para uma tomada de decisão do usuário. Esta tomada de decisão é descrita no tópico seguinte.

Parâmetros binários: Para a regra de mistura de Plocker et al. (1978), o aplicativo abre a caixa de diálogo apresentada na Figura 6. A opção Não, o aplicativo assume que todos os $k_{i j}=1$. A opção Sim abre a caixa de diálogo apresentada na Figura 7 para as especificações dos parâmetros binários. 


\section{Parâmetros de Interação}

○s Parâmetros de Interação São Disponiveis?

(c) $\operatorname{Sim}$

C Năo $(\mathrm{Kij}=1$, para i e $\mathrm{j}=1,2, \ldots, \mathrm{N})$

OK

Cancelar

Figura 6- Disponibilidade dos parâmetros

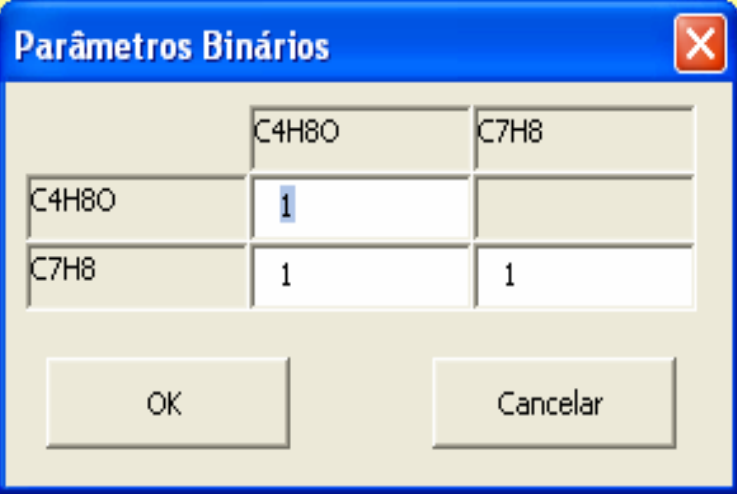

Figura 7 - Especificações dos parâmetros

Especificações da temperatura e pressão: A caixa de diálogo para as especificações da temperatura e da pressão é apresentada na Figura 8.

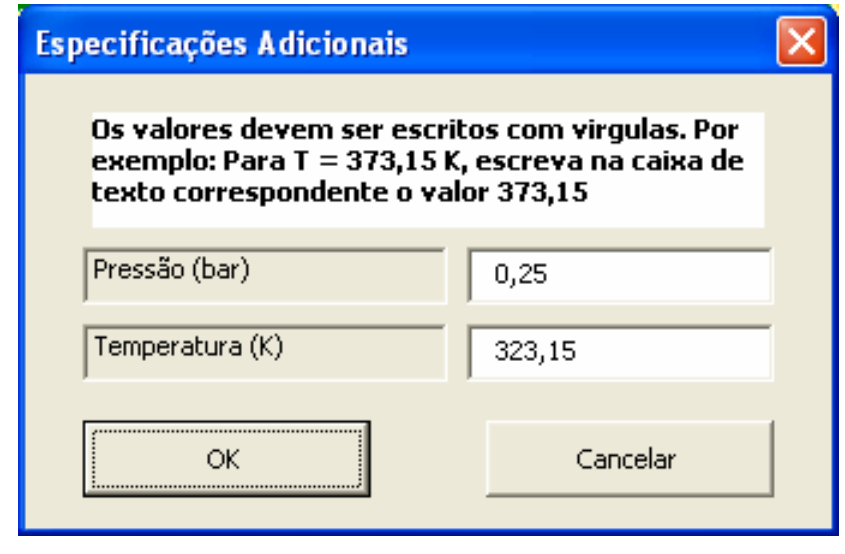

Figura 8 - Especificações temperatura e pressão

\section{RESULTADOS - VALIDAÇÃO DO APLICATIVO}

Vários sistemas foram utilizados para a validação do aplicativo proposto, ou seja, para a verificação e análise dos valores numéricos das fugacidades fornecidos pelos subprogramas codificados. Este trabalho mostra apenas alguns resultados obtidos.

Coeficiente de fugacidade da água: A Figura 9 mostra a comparação entre os valores do coeficiente de fugacidade da água em função da pressão, em várias temperaturas, reportados por Holser (1954) com os estimados através do aplicativo. As propriedades físicas utilizadas são: $T_{c}=$ $647,3 \mathrm{~K}$ e $P_{c}=221,2$ bar com o fator acêntrico estimado através da correlação reportada por Lee e Kesler (1975). Os resultados indicam que a equação de estado proposta por Lee e Kesler (1975) fornecem excelentes resultados nas estimativas do coeficiente de fugacidade da água na faixa de pressão de 1 a 800 bar, em todas as faixas de temperatura, perdendo eficiência a medida que a pressão aumenta. 


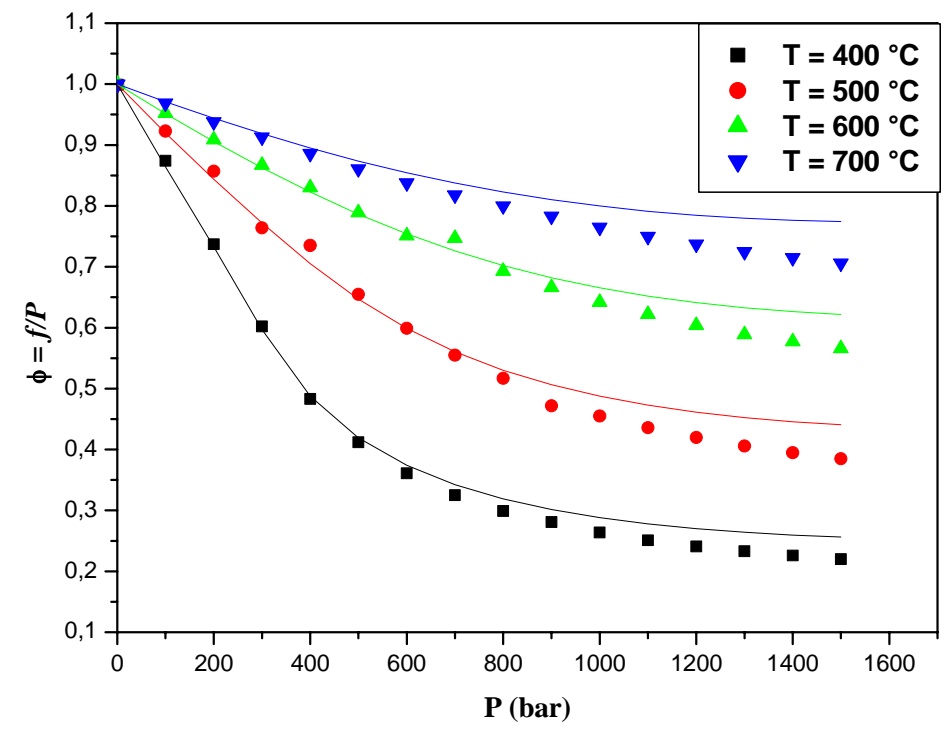

Figura 9 - Valores do coeficiente de fugacidade da água em função da pressão

Sistema amônia (1) - propano (2): A comparação entre os resultados reportados por Antezana e Cheh (1976) com os obtidos através do aplicativo para o sistema amônia (1) - propano (2) é apresentado na Tabela 2. Os fatores acêntricos das espécies são: a) banco de dados: $w_{1}=0,25$ e $w_{2}=$ 0,153 e b) Lee-Kesler: $w_{1}=0,2442$ e $w_{2}=0,1501$. A simbologia empregada é: $y_{1}$ é a fração molar da espécie 1; AC são dados reportados por Antezana e Cheh (1976); Aplic1 são dados obtidos com o fator acêntrico estimado através da correlação de Lee e Kesler (1975) e Aplic2 são dados obtidos com o fator acêntrico experimental (banco de dados).

Tabela 2 - Resultados finais para o sistema amônia (1) - propano (2)

\begin{tabular}{ccccccccc}
\hline \multirow{2}{*}{$\mathrm{T}(\mathrm{K})$} & \multirow{2}{*}{$\mathrm{P}(\mathrm{atm})$} & \multirow{2}{*}{$y_{1}$} & \multicolumn{3}{c}{ Fugacidade (atm) } & \multicolumn{3}{c}{ Coeficiente de fugacidade } \\
\cline { 4 - 8 } & & AC & Aplic1 & Aplic2 & AC & Aplic1 & Aplic2 \\
\hline 327,15 & 19,35 & 0,605 & 10,26 & 10,415 & 10,408 & 0,876 & 0,890 & 0,889 \\
327,45 & 17,64 & 0,648 & 10,23 & 10,276 & 10,270 & 0,895 & 0,899 & 0,898 \\
326,95 & 16,02 & 0,699 & 10,22 & 10,160 & 10,154 & 0,913 & 0,907 & 0,907 \\
327,15 & 11,06 & 1,000 & 10,18 & 10,345 & 10,341 & 0,920 & 0,935 & 0,935 \\
344,45 & 28,74 & 0,814 & 19,82 & 20,130 & 20,115 & 0,847 & 0,860 & 0,860 \\
344,45 & 27,44 & 0,851 & 19,78 & 20,233 & 20,219 & 0,847 & 0,866 & 0,866 \\
344,45 & 22,84 & 1,000 & 19,71 & 20,290 & 20,278 & 0,863 & 0,888 & 0,888 \\
\hline
\end{tabular}

Sistema hidrogênio (1) - propano (2): A comparação entre os resultados reportados por Antezana e Cheh (1975) com os obtidos através do aplicativo para o sistema hidrogênio (1) - propano (2) é apresentada na Tabela 3. As propriedades físicas são: $T_{c 1}=33,2 \mathrm{~K}, T_{c 2}=369,8 \mathrm{~K}, P_{c 1}=13$ bar e $P_{c 2}=42,5$ bar. Os fatores acêntricos das espécies são estimados através da correlação reportada por Lee e Kesler (1975). Na Tabela 3, Aplic1 são resultados obtidos com a regra de mistura de Lee-Kesler e Aplic2 com a regra de mistura de Plocker et al. (1978) com $k_{12}=1,826$. 
Tabela 3 - Resultados finais para o sistema hidrogênio (1) - propano (2) a 391,75 K

\begin{tabular}{rccccccc}
\hline \multirow{2}{*}{ P (atm) } & \multirow{2}{*}{$y_{1}$} & \multicolumn{3}{c}{ Fugacidade (atm) } & \multicolumn{2}{c}{ Coeficiente de fugacidade } \\
\cline { 3 - 7 } & & AC & Aplic1 & Aplic2 & AC & Aplic1 & Aplic2 \\
\hline 32,281 & 0,310 & 11,407 & 11,5917 & 11,4961 & 1,14 & 1,1583 & 1,1488 \\
32,568 & 0,412 & 14,727 & 14,7651 & 14,6680 & 1,098 & 1,1004 & 1,0931 \\
53,593 & 0,275 & 18,767 & 19,8943 & 19,6070 & 1,273 & 1,3499 & 1,3304 \\
53,468 & 0,430 & 26,438 & 26,6757 & 26,4077 & 1,150 & 1,1603 & 1,1486 \\
53,568 & 0,547 & 32,495 & 31,9769 & 31,7701 & 1,109 & 1,0913 & 1,0842 \\
61,913 & 0,300 & 24,824 & 25,4487 & 25,0428 & 1,336 & 1,3701 & 1,3483 \\
67,837 & 0,439 & 35,857 & 35,7701 & 35,8022 & 1,204 & 1,2011 & 1,1865 \\
\hline
\end{tabular}

\section{CONCLUSÃO}

Este trabalho apresenta uma planilha capaz de fornecer com confiabilidade e rapidez as estimativas de fugacidades de espécies puras, mistura e de componentes de uma mistura. Deve ser observado que a confiabilidade dessas estimativas está associada à eficiência da equação de estado de Lee-Kesler e não por erros numéricos ou erros de codificações dos subprogramas VBA.

\section{REFERÊNCIA}

ANTEZANA F. J., CHEH, H. Y. Component Fugacities in Hydrogen-Ammonia-Propane Mixtures. I. The Fugacity of Hydrogen. Ind. Eng. Chem. Fundam., Vol. 14, No. 3, 1975.

ANTEZANA F. J., CHEH, H. Y. Component Fugacities in Hydrogen-Ammonia-Propane Mixtures. II. The Fugacity of Ammonia. Ind. Eng. Chem. Fundam., Vol. 15, No. 2, 1976.

ASSAEL, M. J., TRUSLER, J. P. M; TSOLAKIS, T. F. Thermophysical Properties of Fluids An Introduction to their Prediction. Imperial College Press, 1998.

COSTA, N. A. Modelagem Matemática da Termodinâmica Química - Fugacidade/Coeficiente de Fugacidade. Volume IV. Documento pessoal, 2014.

LEE, B. I., KESLER, M. G. A Generalized Thermodynamic Correlation Based on ThreeParameter Corresponding States. AIChE Journal. Vol. 21, 510, 1975.

REID, R. C; PRAUSNITZ, J. M; POLING, B. E. The Properties of Gases \& Liquids. Fourth Edition. McGraw-Hill Book Company, 1988.

PLOCKER, U; KNAPP, H; PRAUSNITZ, J. M; Calculation of High-Pressure Vapor-Liquid Equilibria from a Corresponding-States Correlation with Emphasis on Asymmetric Mixtures. Ind. Eng. Chem. Process Des. Dev., Vol. 17, No 3, 324-332, 1978.

POLING, B. E; PRAUSNITZ, J. M; O'CONNEL, J. P. The Properties of Gases and Liquids. Fifth Edition. McGraw-Hill, 2000.

SMITH, J. M; Van Ness, H. C; ABBOTT, M. M. Introdução à Termodinâmica da Engenharia Química. Quinta Edição. LTC; 2007. 